\title{
Prima della tragedia. Militari italiani a Cefalonia e a Corfú
}

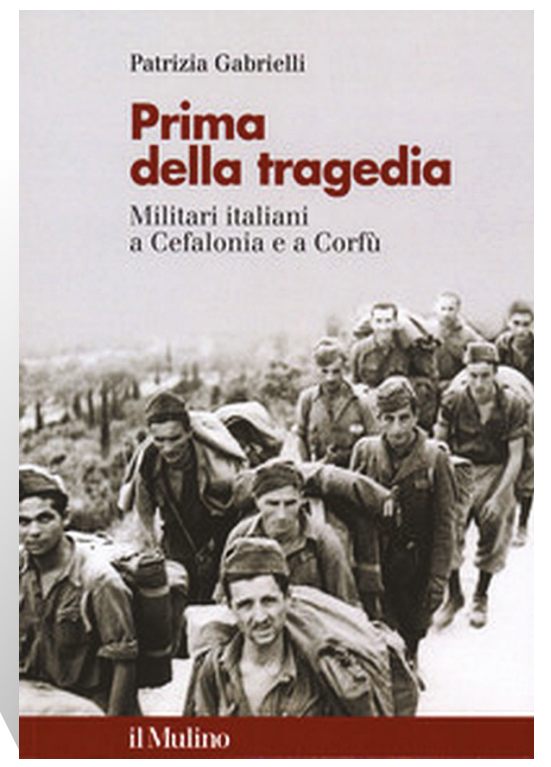

FICHA BIBLIOGRÁFICA

Patrizia Gabrielli, Prima della tragedia. Militari italiani a Cefalonia e a Corfú, Bologna, il Mulino, 2020, 173 páginas, ISBN, 9788815275578.

\section{Laura Branciforte I Universidad Carlos III de Madrid}

QUESTO LIBRO FORNISCE UN CONTRIBUTO NUOVO a una parte della storia spesso ancora trascurata: la guerra contro la Grecia e le occupazioni balcaniche, che soltanto recententemente, sono state oggetto di un rinnovato interesse storiografico. A Cefalonia e a Corfù i militari tedeschi trucidarono alcune migliaia di militari italiani che non si erano arresi, queste due isole ioniche divennero, dunque, i luoghi tristemente conosciuti di un 'massacro indiscriminato'. Le vicende dell' isolamento e della strage nazista nei giorni tra l' 8 e il 22 settembre del 1943 vengono ora rilette da Patrizia Gabrielli attraverso una rigorosa e partecipata ricostruzione basata su lettere inviate dai militari alle famiglie e viceversa, prima di quel tragico epilogo.

Il libro di Gabrielli esplora i sentimenti, il cordoglio, le memorie spezzate e, infine, le scelte e le incertezze vissute dai soldati e dagli ufficiali prima del momento di cedere le armi ai Tedeschi dopo l'armistizio. Nella ricerca sulle vicende riguardanti gli uomini che combat- 
terono a Corfù e a Cefalonia la storica supera le interpretazioni e le visioni, spesso polarizzate, sull'azione dei soldati italiani, né eroi né martiri ${ }^{1}$ e dei quali non tralascia di sottolineare la dignità e il senso dell'onore militare come parte dei sentimenti espressi dai sopravvisuti, spesso giudicati come traditori.

Il tema, malgrado sia già stato oggetto, di importanti ricerche nell'ambito della storiografia italiana (Giorgio Rochat, Gerhard Schreiber, Mario Montanari, Nicola Labanca e altri ancora) rappresenta una novità per la scelta delle fonti, l'epistolografia. L'Autrice, che si è cimentata in anteriori pubblicazioni (fra cui Scenari di guerre, parole di donna. Diari e memorie nell'Italia della Seconda guerra mondiale, 2007) con l'uso di fonti memorialistiche e diaristiche, sceglie ora le lettere, ovvero una fonte ancora più diretta e immediata, un documento grazie al quale, come lei stessa sottolinea, si rielabora il dialogo «tra la memoria individuale e quella collettiva» e si dà vita ad una «autobiografia collettiva» (p. 7). Le guerre, infatti, hanno reso questa pratica di scrittura molto più diffusa: queste fonti hanno in parte colmato il vuoto e la separazione dagli affetti; ricordo qui un caso a me caro, le missive scritte durante la prigionia in India alla moglie e ai figli da mio nonno ${ }^{2}$. Questa ricerca mi è risultata quindi ancora più prossima tanto per i vincoli emotivi che mi legano a questo tipo di documento, quanto come studiosa che ne riconosce l'alto valore testimoniale.

Il carattere intimo della fonte non priva, ad ogni modo, di rigore al volume di Gabrielli in cui si constata la ricchezza e la varietà dei fondi e degli archivi consultati, tra cui spicca soprattutto l'Archivio dell' Istituto storico autonomo della Resistenza dei militari italiani all'estero (Isaremi) al quale l'Autrice dedica, direi quasi come un omaggio alla memoria e all' importanza e al legame con le sue fonti, le pagine di chiusura, le "Note d'Archivio". Le espistole sono, inoltre, sempre accompagnate da un'analisi e da approfondimenti che collocano queste fonti private, soggettive, nel contesto storico e politico preciso. La pressante realtà del fascismo accompagna il lettore in un crescendo che raggiunge il suo punto algido quando il ritmo inclanzante delle lettere - consci, noi tutti, del finale dei fatti di Corfù e di Cefalonia conduce a ciò che nel titolo annuncia il "dopo tragedia" e si attendeva con timore. La parte più struggente del libro inizia l' 8 settembre, dopo i 45 giorni trascorsi dal 26 luglio dalla caduta del fascismo. Un 8 settembre la cui assenza di significato viene riassunta in modo lapidario dal soldato Pietro Visintini, che scrive alla famiglia: «l'armistizio invece di portare la pace ha portato la guerra. Si combatte contro i tedeschi...» (p.136). Il valore di questa lettera che, in poche linee, sintetizza il dramma della storia e delle conseguenze di decisioni e scelte sbagliate conferma, ancora una volta, il valore stesso dei libri di memorie, i quali riscostruiscono e fanno capire le vicende storiche a partire dai soggetti in carne e ossa. Un libro, quello di Gabrielli, che fino alla fine getta luce sulle sorti di questi uomini, donne e bambini che nel fronte di guerra e nel fronte interno soffrirono la guerra e le conseguenze di una battaglia che dal 15 al 22 settembre decimò gli italiani a Corfù e a Cefalonia.

1. C. Brezzi (a cura di), Né eroi, né martiri. Soltanto soldadi. La divisione Aqui a Cefalonia e Corfu', settembre 1943, Bologna, 2014.

2. G. Branciforte, Diario della prigionia in Italia e ricordi vari, Roma, 2008. 
A questo punto le lettere diminuiscono e, nelle poche che l'Autrice ha potuto offrire al lettore, prevalgono il silenzio e lo smarrimeto; le terribili conseguenze della resistenza al disarmo a Corfù e a Cafalonia, la strage tedesca durata una settimana interminabile, la vittoria infine della Wehrmacht sulla Divisione Acqui, sono il tragico epilogo. Prende piede lo smarrimento e l'empatia del lettore con la sue fonte, della quale ha imparato i nomi e conosciuto le vite, reali, non fittizie, come quella di Erminio Bolpin, con il quale ha gioito e sofferto e, infine, vissuto il momento in cui una lettera di un familiare gli comunica che è diventato padre, notizia che genera tristezza per una paternità che non potrà sperimentare (p. 113).

Infine, non perché meno importante, vorrei sottolineare la sensibilità della scrittura di una storica di genere, che mette in evidenza la rilevanza dell'operato delle donne come «intermediare» tra l'ambito privato, cioè la ricezione delle lettere, e quello pubblico, di trasmissione delle notizie dal fronte bellico. L'arrivo di una lettera in tempi di guerra non resiste alla separazione degli scenari pubblico/privato. Seppur lontane dai fronti di guerra, le donne, le mogli, le madri, le sorelle, le bambine sono presenti in questo libro. Sarebbe miope non riconoscere, da lettrice delle pubblicazioni di Gabrielli, l'attenzione verso la ricostruzione della memoria dei bambini, come mostra il libro La guerra è l'unico pensiero che ci domina tutti. Bambine, bambini, adolescenti nella Grande guerra. Come sottolinea la stessa Autrice, i bambini e le bambine «sono oramai diventati uomini e donne, soggetti attivi, non solo vittime, le cui pagine sembrano ricomporre il trauma della perdita» (p. 23).

Come spesso accade quando si affrontano temi di ricerca nuovi la Autrice lascia inesplorato un aspetto al quale ad ogni modo allude, ammettendo la scarsezza delle fonti, ovvero l'incontro degli Italiani con la popolazione locale: una storia bella, fatta non soltanto del rifiuto, conosciuto e reale, dell' invasore italiano, ma anche, come ricorda la storica, del maternage (p. 153) e della solidarietà femminile che prima della tragedia riuscì a dare protezione e salvare la vita di giovani spesso ignari del loro vero nemico. Fare luce su un aspetto spesso trivializzato, quello dell' incontro tra gli Italiani e le donne che divennero le loro amiche, mamme, compagne ed anche amanti, potrebbe dunque essere, come auspichiamo, il tema di una futura ricerca di Patrizia Gabrielli. 